\title{
Risk Factors for Doxorubicin-Induced Serious Hyperglycaemia-Related Adverse Drug Reactions
}

Jincheng Yang (D) - Yu Wang • Kang Liu • Wen Yang •

Jianying Zhang

Received: June 12, 2019 / Published online: August 19, 2019

(c) The Author(s) 2019

\section{ABSTRACT}

Introduction: Mortality rates from various cancer types are higher in patients with hyperglycaemia-associated pathologies. Among antineoplastics for treating pancreatic carcinoma, doxorubicin was found to be top of the list for inducing hyperglycaemia-related adverse drug reactions.

Methods: Individual case safety reports of doxorubicin-induced hyperglycaemia-related adverse drug reactions (HG-ADRs) submitted during the period 2000-2017 were extracted from VigiBase ${ }^{\circledR}$. Factors influencing outcome seriousness in these cases were analyzed using bivariate correlation and logistical regression. Age was stratified into five groups.

Results: Among the 558 cases with high blood glucose included in this study, with an average patient age of $55.9 \pm 14.3$ years, $71.1 \%$ had

Enhanced Digital Features To view enhanced digital features for this article go to https://doi.org/10.6084/ m9.figshare.9116393.

J. Yang $(\bowtie) \cdot$ Y. Wang $\cdot$ K. Liu · W. Yang · J. Zhang Department of Pharmacy, National Cancer Center/ National Clinical Research Center for Cancer/ Cancer Hospital, Chinese Academy of Medical Sciences and Peking Union Medical College, Beijing, China

e-mail: Yangjc@cicams.ac.cn serious outcomes and $10.9 \%$ died. Three factors-died, age, and gender-were all found to be significantly correlated with outcome seriousness $(P<0.01)$, while five factors-year reported, ADR duration, latency, treatment duration, and dosage-showed no correlation with outcome seriousness $(P>0.05)$. Identical conclusions were reached upon analyzing only the cases that did not involve corticosteroid use. Conclusion: Age and gender are risk factors for doxorubicin-induced serious HG-ADRs; males and older patients are more likely to suffer a serious outcome following such a reaction.

Keywords: ADR; Adverse drug reaction; Doxorubicin; Hyperglycaemia; VigiBase

\section{INTRODUCTION}

Hyperglycaemia (high blood glucose) and its effects on remission and survival in cancer patients have attracted considerable clinical attention. Hyperglycaemia, defined as two consecutive glucose values of $\geq 250 \mathrm{mg} / \mathrm{dL}$ $(13.89 \mathrm{mmol} / \mathrm{L})$, is common in patients with hematologic malignancies [1]. Hyperglycaemia occurs in approximately $10-30 \%$ of all patients undergoing chemotherapy [2].

Hyperglycaemia also heightens the risk for the development and progression of cancer, affects diagnostic imaging studies, attenuates the immune system, and increases resistance to 
chemotherapy [3]. The cancer-specific death rate tends to rise with mean fasting glucose level. Hyperglycaemia has been proposed as one of the primary underlying mechanisms for the increased mortality rate observed for diabetic cancer patients [4]. Uncontrolled high glucose has recently been linked to an increased rate of surgical site infection after colorectal and general surgery in both diabetic and nondiabetic patients [5]. Hyperglycaemia is also a hallmark of both type 1 diabetes mellitus (T1DM) and type 2 diabetes mellitus (T2DM). Recent evidence strongly suggests that prolonged exposure to hyperglycaemia can epigenetically modify gene expression profiles in human cells, and that this effect is sustained even after glycemic control is achieved therapeutically (this phenomenon is called hyperglycemic memory) [6]. In cancer patients, hyperglycaemia frequently occurs independently of the diagnosis of diabetes [3].

Doxorubicin is an anthracycline antibiotic and antineoplastic that is widely used in breast cancer, lung cancer, acute leukemia, and malignant lymphoma. It is also associated with adverse drug reactions (ADRs) such as bone marrow suppression, ulcer, cardiotoxicity, gastrointestinal reaction, liver and kidney dysfunction, and alopecia. We previously noted that, among antineoplastics used to treat pancreatic carcinoma, doxorubicin is top of the list for Hyperglycaemia-related ADRs (HG-ADRs) [7], as well as HG-ADR overall distribution of doxorubicin [8]. In order to determine the risk factors for hyperglycaemia, we investigated doxorubicininduced HG-ADR using the WHO Global Pharmacovigilance Database (VigiBase ${ }^{\circledR}$ ).

\section{METHODS}

\section{Data Source}

All data presented herein were sourced from VigiBase $^{\circledR}$, the World Health Organization's (WHO) global database for ADRs, maintained by the Uppsala Monitoring Centre (UMC). The $\mathrm{UMC}$ is the WHO Collaborating Centre for International Drug Monitoring, which provides scientific leadership and operative support to the WHO Program for International Drug
Monitoring (www.who-umc.org). This database contains reports of suspected ADRs, called individual case safety reports (ICSRs), that have been collected by national drug authorities in over 110 countries and span more than 100,000 medicinal products.

\section{Data Collection}

Substance: doxorubicin. Drug involvement: suspected/interacting. Terminology: MedDRA. Organization type: non-commercial/academia. Reactions: all. Country: all. Years: from 2000 to 2017.

\section{Inclusion Criteria}

Medical Dictionary for Regulatory Activities (MedDRA) preferred terms (PTs): hyperglycaemia, blood glucose increased, diabetes mellitus, type 2 diabetes mellitus, type 1 diabetes mellitus, diabetes insipidus, diabetes mellitus inadequate control, gestational diabetes, hyperglycemic hyperosmolar nonketotic syndrome.

Drug names (preferred base name or reported drug): doxorubicin, doxorubicina, doxorubicine, lipodox, liposomal doxorubicin, myocet, caelyx, doxil, hydroxydaunorubicin, adriblastine, adriamycin.

\section{Exclusion Criteria}

Items without hyperglycaemia-related MedDRA PTs or without doxorubicin-related drug names.

Corticosteroids: dexamethasone, prednisone, methylprednisone, prednisolone, hydrocortisone, cortisone.

\section{Data Analysis}

Eight factors-year reported, died, age, gender, ADR duration, latency, treatment duration, and dosage-were selected for correlation analysis. All data were analyzed using SPSS version 25.0 (IBM Corp., Armonk, NY, USA). Pictures were drawn with Microsoft Excel Professional 2016. A $P$ value of $<0.05$ was considered to be statistically significant. 


\section{Compliance with Ethics Guidelines}

This study is based on data mining and interpretation of the largest database for drug safety in the world (the WHO-UMC VigiBase ${ }^{\circledR}$ ) and does not contain any studies with human participants or animals performed by any of the authors. Therefore, no ethical review board approval was required.

\section{RESULTS}

Reports for a total of 558 cases with an average patient age of $55.9 \pm 14.3$ years were collected, spanning the period from 2000 to 2017 . Table 1

Table 1 Hyperglycaemia-related ADRs

\begin{tabular}{lcc}
\hline MedDRA PT name & $\begin{array}{l}\text { Number of } \\
\text { cases }(\boldsymbol{N})\end{array}$ & $\begin{array}{l}\text { Proportion } \\
(\%)\end{array}$ \\
\hline Hyperglycaemia & 369 & 66.1 \\
Blood glucose increased & 94 & 16.8 \\
Diabetes mellitus & 40 & 7.2 \\
Type 2 diabetes mellitus & 31 & 5.6 \\
Diabetes mellitus & 4 & 0.7 \\
$\quad$ aggravated & & \\
Diabetes insipidus & 3 & 0.5 \\
Diabetes mellitus & 3 & 0.5 \\
inadequate control & & \\
Gestational diabetes & 3 & 0.5 \\
Hyperglycaemia NOS & 3 & 0.5 \\
Diabetes mellitus insulin- & 2 & 0.4 \\
dependent & & 0.4 \\
Type 1 diabetes mellitus & 2 & 0.4 \\
Worsening of diabetes & 2 & 0.2 \\
Diabetes mellitus non- & 1 & 100.0 \\
insulin-dependent & & \\
Hyperosmolar & 1 & \\
hyperglycemic state & & \\
Total & 558 & \\
\hline & & \\
\hline
\end{tabular}

shows that cases with the MedDRA PTs "hyperglycaemia" and "blood glucose increased" comprised $83.7 \%$ of all cases, and cases with diabetes-related MedDRA PTs comprised 16.3\%. The demographic characteristics of the cases are summarized in Table 2 . The ratio of male to female patients in the case reports was around 1:2. Serious HG-ADRs comprised 71.1\% (382/ 537 ) of all cases for which the seriousness of the ADR was recorded, and died cases comprised $10.9 \%(61 / 558)$ of all cases. Note that some of the reports did not include data on all eight factors, which is why the number of relevant cases for each factor is smaller than the total number of cases (558).

Fig. 1 shows that the main effects of the HGADRs were prolonged hospitalization (29.6\%, 165 cases) and death or a life-threatening state (15.0\%, 84 cases).

The number of relevant reports in 2015 was unusually high; this was because 148 of the reports in that year derived from studies (as opposed to being unrelated to studies, i.e., reports written "spontaneously"), which was a much higher number of study-related reports than seen for any other year. To avoid statistical bias, we only included the non-study-related cases (30) from 2015 in the subsequent correlation analysis. It should be noted that even if we include the study-related reports from 2015, the number of "spontaneous" (non-study-related) reports is still greater than the number of study-related reports submitted during 2000-2017 (309 vs 238, Fig. 2).

The correlations of the eight factors considered with serious outcome were analyzed, and the results are summarized in Table 3 . As corticosteroids are commonly used in cancer treatment, we also analyzed only the cases in which corticosteroids were not used. Three factorsdied, age, and gender-showed very strong correlations with serious outcome $(P<0.01)$, whereas year reported, ADR duration, latency, treatment duration, and dosage were uncorrelated with serious outcome $(P>0.05)$. However, we did not include the factor died in the subsequent analysis of factors that influence the seriousness of the HG-ADR, as death is a potential severe consequence of a serious HG-ADR rather than a factor that impacts on $\mathrm{ADR}$ 
Table 2 Main characteristics of the cases

\begin{tabular}{|c|c|c|c|c|}
\hline Factor & Relevant data & $\begin{array}{l}\text { Total number of } \\
\text { relevant cases }\end{array}$ & Mean & Standard deviation \\
\hline Year reported & Range: $2000-2017$ & 558 & - & - \\
\hline Serious ADR & $\mathrm{Y} / \mathrm{N}^{\mathrm{a}}$ (cases): $382 / 155$ & 537 & - & - \\
\hline \multirow[t]{2}{*}{ Age } & \multirow[t]{2}{*}{ Range (years): 13.00-95.34 } & \multirow[t]{2}{*}{344} & $57.28(\mathrm{M})^{\mathrm{e}}$ & $16.90(\mathrm{M})$ \\
\hline & & & $55.04(\mathrm{~F})$ & $12.94(\mathrm{~F})$ \\
\hline Gender & $M / F^{e}: 135 / 309$ & 444 & - & - \\
\hline ADR duration ${ }^{b}$ & Range (days): 0-139 & 40 & 22.93 & 33.90 \\
\hline Latency $^{\mathrm{c}}$ & Range (days): 0-155 & 51 & 26.41 & 34.73 \\
\hline Treat duration $^{\mathrm{d}}$ & Range (days): 0-365 & 109 & 40.82 & 66.58 \\
\hline Post-treatment $\mathrm{ADR}^{\mathrm{f}}$ & Range (days): 0-110 & 9 & 32.33 & 40.62 \\
\hline Died & $\mathrm{Y} / \mathrm{N}^{\mathrm{a}}$ (cases): 61/497 & 558 & - & - \\
\hline
\end{tabular}

a $Y$ yes, $N$ no

b Number of days from onset date (date of start of reaction) to resolution date (date of end of reaction)

c Number of days from start date (date of starting drug) to onset date (date of start of reaction)

d Number of days from start date (date of starting drug) to stop date (date of last administration of drug)

e $F$ female, $M$ male

${ }^{\mathrm{f}}$ Number of days from stop date (date of last administration of drug) to resolution date (date of end of reaction)

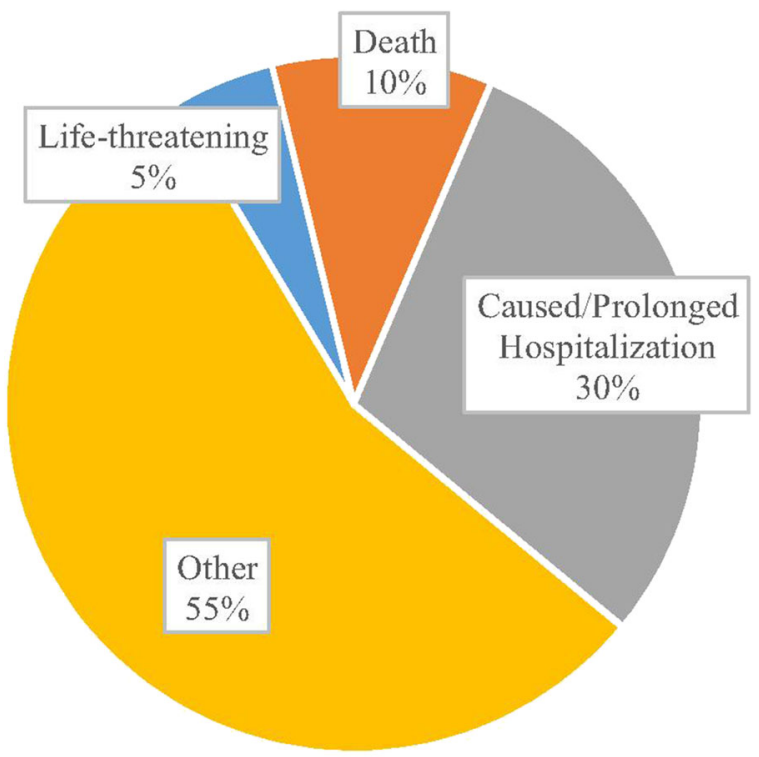

Fig. 1 Categorization of the cases based on HG-ADR seriousness

seriousness. After performing paired comparisons, we planned to determine risk factor(s) for treatment duration using Cox regression, but the majority of the case reports did not include the relevant data.

Next, we categorized the cases into five patient age groups $(0-44,45-54,55-64,65-74$, and 75-99 years) following the International Cancer Survival Standards for age standardization of survival [9]. The correlation of each age group with serious outcome is shown in Table 4 . The 0-44, 55-64, and 65-74 groups were found to be significantly correlated with serious HGADRs.

According to Table 3, age and gender were significantly correlated with serious outcome, so these two factors were then analyzed through logistic regression. The results, shown in Table 5, indicated that these two factors significantly influenced outcome seriousness (when these factors were removed from the equation, there was significant change, $P<0.01$ ). The correlations presented in Table 5 are similar to those given for age and gender in Table 3: age is positively correlated and gender is negatively correlated with outcome seriousness. 


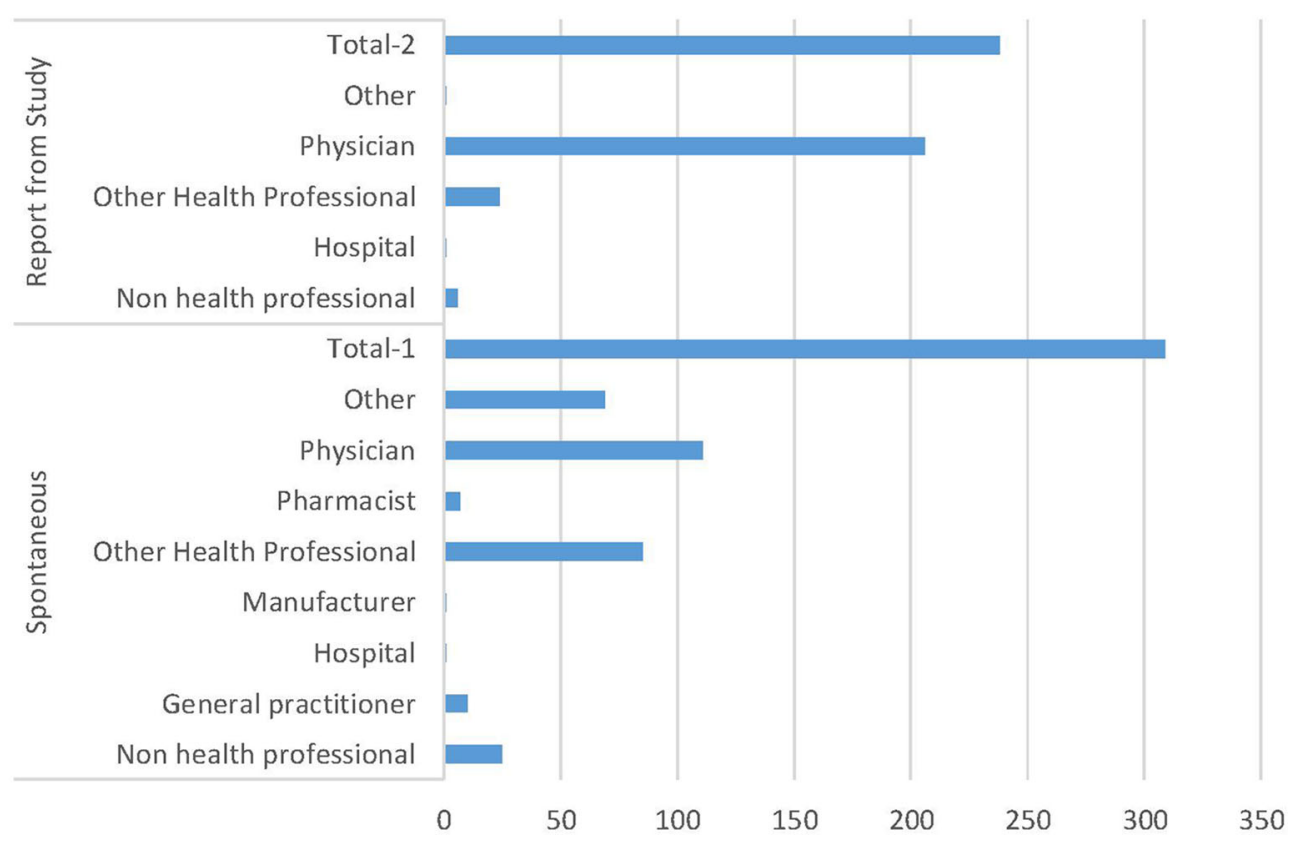

Fig. 2 Categorization of the cases based on report type (study-related or "spontaneous") and report notifier

\section{DISCUSSION}

Doxorubicin is an antineoplastic that is widely used to treat a variety of solid tumors. Diabetic patients are at increased risk of developing various types of cancer, and patients with hyperglycaemia-associated pathologies show higher rates of mortality from a range of cancer types. Hyperglycaemia promotes chemoresistance (reduced cell death) by reverting the doxorubicininduced increased percentage of cells in the subG1 peak (caused by cell cycle arrest in the G2/M phase) [10]. Another study showed that doxorubicin-induced hyperglycaemia is mediated via inhibition of AMPK signaling in skeletal muscle [11].

Knowledge that hyperglycaemia leads to an increased length of stay in hospital, delays wound healing, and increases infection and mortality rates should encourage healthcare providers to monitor for its occurrence and manage it appropriately [12]. Our study evaluated data relating to doxorubicin-induced HGADRs from the international pharmacovigilance database VigiBase ${ }^{\circledR}$ and thus offers insight into the HG-ADRs that occur in doxorubicin-using patients.
Factors contributing to hyperglycaemia in cancer patients include physiologic stress, glycemic status (glucose intolerance, diagnosed with diabetes, diagnosed with pre-diabetes, or unknown diabetes status), administration of glucocorticoids, and changes in nutrition and activity [3]. Cancer and diabetes mellitus share common risk factors: older age, male sex, obesity, lack of physical activity, a high-calorie diet, tobacco smoking, and insulin resistance, all of which can lead to hyperglycaemia. In this study, we identified two risk factors for serious HG-ADRs in patients: age and gender, and one severe consequence of HG-ADRs: death.

Table 2 shows that there were twice as many case reports for female patients than for male patients, which may be explained by the fact that doxorubicin is frequently used in breast cancer. This table also shows that 61 of the patients among all of the cases retrieved died, while Fig. 1 includes 57 cases that ended in death and 27 cases in which the patient was in a life-threatening state. Note that Table 2 shows data on the final outcomes of the patients, whereas Fig. 1 presents the cases categorized according to ADR seriousness. However, both Table 2 and Fig. 1 highlight the severe 


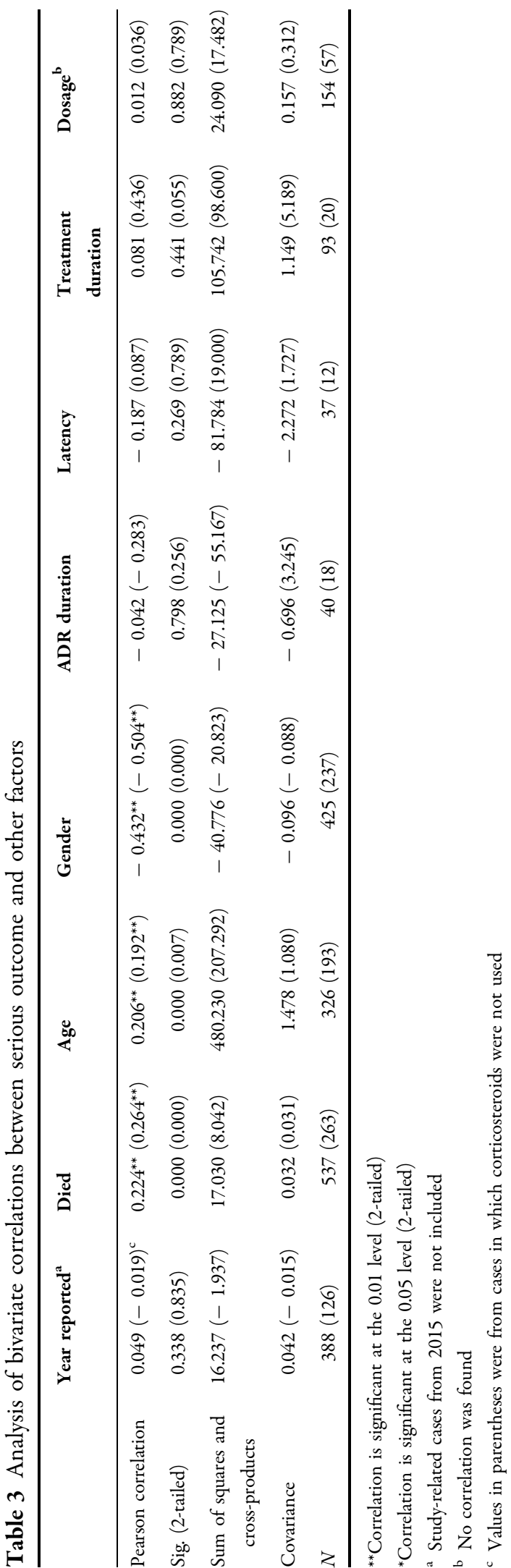

Table 4 Bivariate correlations of five patient age groups with serious outcome

\begin{tabular}{lll}
\hline $\begin{array}{l}\text { Age group } \\
\text { (years) }\end{array}$ & $\begin{array}{l}\text { Analytical } \\
\text { parameter }\end{array}$ & Values \\
\hline $0-44$ & Pearson & $-0.503^{* *}$ \\
& correlation & $\left(-0.436^{* *}\right)^{\mathrm{a}}$ \\
& Sig. (2-tailed) & $0.000(0.002)$ \\
& $N$ & $71(50)$ \\
$45-54$ & Pearson & $-0.087(-0.127)$ \\
& correlation & \\
& Sig. (2-tailed) & $0.465(0.375)$ \\
& $N$ & $73(51)$ \\
$55-64$ & Pearson & $-0.340^{* *}$ \\
& correlation & $\left(-0.346^{* *}\right)$ \\
& Sig. (2-tailed) & $0.001(0.006)$ \\
& $N$ & $91(61)$ \\
$65-74$ & Pearson & $0.357^{* *}\left(0.716^{* *}\right)$ \\
& correlation & \\
& Sig. (2-tailed) & $0.002(0.000)$ \\
& $N$ & $71(28)$ \\
$75-99$ & Pearson & $0.085\left(1.000^{* *}\right)$ \\
& correlation & \\
& Sig. (2-tailed) & $0.722(0.005)$ \\
& $N$ & $20(3)$ \\
\hline
\end{tabular}

${ }^{* *}$ Correlation is significant at the 0.01 level (2-tailed)

${ }^{a}$ Values in parentheses refer to an analysis focusing on cases in which corticosteroids were not used

consequences of HG-ADRs. Figure 2 shows that physicians provided most of the study-related and "spontaneous" (non-study-related) case reports, indicating that the issue of HG-ADRs induced by doxorubicin use has been largely overlooked by other health professionals.

Table 3 shows that the factors died and age were positively correlated with serious outcome, demonstrating that some HG-ADRs ended in death and that HG-ADR seriousness increased with age. As implied by the predominance of female patients among the cases considered, we also discerned a correlation of serious outcome 
Table 5 Results from logistic regressions of age and gender against outcome seriousness

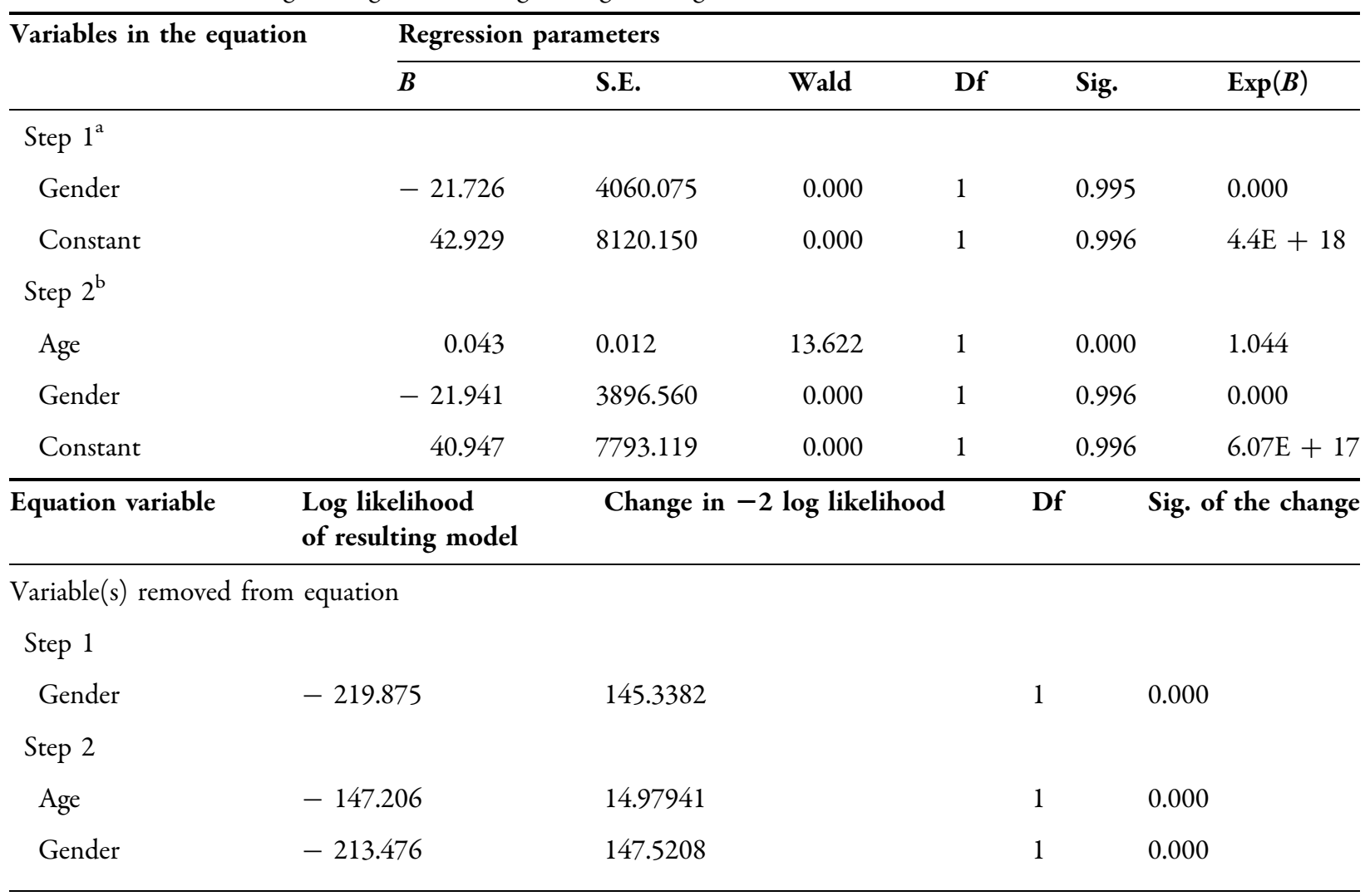

Sig. significance

a Variables included in step 1: gender

b Variables included in step 2: age

with female gender. Meanwhile, our analysis also indicated that ADR duration, latency, treatment duration, and dosage were not correlated with doxorubicin-induced HG-ADR seriousness $(P>0.05)$, suggesting that HG-ADRs are probably type II ADRs (they are not dependent on drug dosage). However, this must be checked by analyzing more data on drug-induced HG-ADRs.

Although age showed a positive correlation with serious outcome overall (see Table 3), different age groups showed differences in their correlations with serious outcome: negative correlations were seen for the 0-44 and 55-64 groups, and a positive correlation was seen for the 65-74 group (see Table 4). We also applied age stratification in our logistic regression and obtained the same correlations (these results are not presented in the present article) as shown in Table 4.
In addition, as blood glucose levels of cancer patients are greatly influenced by the use of drugs such as corticosteroids, we analyzed the cases in which corticosteroids were not taken separately from those cases in which corticosteroids were taken. Among all of the cases for which corticosteroid usage or nonusage was reported, 43.6\% (234/537) involved corticosteroid use. $9.0 \%(14 / 155)$ of the corticosteroidusing patients had non-serious HG-ADRs, whereas 55.4\% (212/382) had serious outcomes. Analysis $\left(\chi^{2}<0.01\right)$ indicated that corticosteroid use was correlated with serious outcome, highlighting that clinical attention should be directed to this issue. Upon extracting and analyzing the cases with no corticosteroid use, we found identical correlations to those seen for all of the doxorubicin-induced HG-ADR cases (see Tables 3 and 4). In Table 4, the 75-99 age group shows a positive correlation with serious 
outcome; however, this correlation was based on only 3 cases. As well as an issue with a low number of cases in some correlation analyses, our study also did not consider the potential effects of other drugs such as monoclonal antibodies (mAbs) on blood glucose. Serious outcomes were reported in 27 of 29 insulin-using cases; maybe insulin injections were needed in those cases to resolve the hyperglycaemia.

In order to check whether the hyperglycaemia subsided after doxorubicin was stopped, data on post-treatment ADR duration (i.e., number of days from the last administration of the drug to the end of the reaction) are listed in Table 2. However, these results are based on data from only 9 cases, all of which involved corticosteroid use.

A randomized controlled clinical trial demonstrated the effectiveness of onion, a functional food, at ameliorating hyperglycaemia and insulin resistance in breast cancer patients undergoing doxorubicin-based chemotherapy [13]. Before standardized guidelines for treating hyperglycaemia can be devised, more information on HG in cancer patients is needed, such as high-risk populations, incidence rates, and the time, degree, and duration of glucose elevation. Also, there is an urgent need to publish a recommendation that HG should be monitored and prevented in such patients.

\section{LIMITATION}

The volume of ICSRs (case reports) available for a particular medicinal product may be influenced by the popularity of and publicity for the product, as well as the nature of the reactions induced by the product. Also, VigiBase ${ }^{\circledR}$ is only a repository of ICSRs, and data collection for this repository differs between countries due to differing national legislation and policies. Thus, data comprehensiveness and integrity restricted deeper analysis of doxorubicin-induced HGADRs here. Future studies should utilize more comprehensive patient information collection, as this would facilitate the identification of the exact effects of HG-ADRs for different areas, populations, and treatment courses.

\section{CONCLUSION}

Among the doxorubicin-induced HG-ADR cases analyzed in this work, there were substantially more cases involving female patients than male patients, and the likelihood of a serious outcome was higher in old age, although each age group exhibited its own correlation with serious HG-ADRs. These two groups (males and the elderly) should be monitored more closely during doxorubicin treatment, especially if they are using corticosteroids. More investigations of other drugs are needed to identify whether drug-induced hyperglycaemia is a non-dosedependent adverse reaction.

\section{ACKNOWLEDGEMENTS}

Funding. The work and the journal's Rapid Service Fee were supported by the National Natural Science Foundation of China (no. 81603218).

Authorship. All named authors meet the International Committee of Medical Journal Editors (ICMJE) criteria for authorship for this article, take responsibility for the integrity of the work as a whole, and have given their approval for this version to be published.

Compliance with Ethics Guidelines. This study is based on data mining and interpretation of the largest database for drug safety in the world (the WHO-UMC VigiBase ${ }^{\circledR}$ ) and does not contain any studies with human participants or animals performed by any of the authors. Therefore, no ethical review board approval was required.

Disclosures. The authors Jincheng Yang, Yu Wang, Kang Liu, Wen Yang, and Jianying Zhang have no conflicts of interest to disclose that are directly relevant to the content of this work.

Data Availability. The datasets generated and/or analyzed during the current study are 
available in the VigiBase ${ }^{\circledR}$ repository at http:// www.who-umc.org.

Open Access. This article is distributed under the terms of the Creative Commons Attribution-NonCommercial 4.0 International License (http://creativecommons.org/licenses/ by-nc/4.0/), which permits any noncommercial use, distribution, and reproduction in any medium, provided you give appropriate credit to the original author(s) and the source, provide a link to the Creative Commons license, and indicate if changes were made.

\section{REFERENCES}

1. Brady V, et al. Safe and effective dosing of basalbolus insulin in patients receiving high-dose steroids for hyper-cyclophosphamide, doxorubicin, vincristine, and dexamethasone chemotherapy. Diabetes Technol Ther. 2014;16(12):874-9.

2. Hwangbo Y, Lee E. Acute hyperglycemia associated with anti-cancer medication. Endocrinol Metab (Seoul). 2017;32(1):23-9.

3. Storey S, Von Ah D, Hammer M. Measurement of hyperglycemia and impact on health outcomes in people with cancer: challenges and opportunities. Oncol Nurs Forum. 2017;44(4):E141-51.

4. Vasconcelos-Dos-Santos A, et al. Hyperglycemia and aberrant O-GlcNAcylation: contributions to tumor progression. J Bioenerg Biomembr. 2018;50(3):175-87.

5. Fiorillo C, et al. Postoperative hyperglycemia in nondiabetic patients after gastric surgery for cancer: perioperative outcomes. Gastric Cancer. 2017;20(3):536-42.

6. Lee C, An D, Park J. Hyperglycemic memory in metabolism and cancer. Horm Mol Biol Clin Investig. 2016;26(2):77-85.

7. Yang J, et al. Glycaemic adverse drug reactions from anti-neoplastics used in treating pancreatic cancer. Niger J Clin Pract. 2017;20(11):1422-7.

8. Yang J, Yang J. Hyperglycemic ADR distribution of doxorubicin from VigiBase. Am J Ther. 2019;26(3):e428-30.

9. Zeng $\mathrm{H}$, et al. Changing cancer survival in China during 2003-2015: a pooled analysis of 17 population-based cancer registries. Lancet Glob Health. 2018;6(5):e555-67.

10. Bergandi $\mathrm{L}$, et al. Hyperglycemia promotes chemoresistance through the reduction of the mitochondrial DNA damage the Bax/Bcl-2 and Bax/ $\mathrm{Bcl}-\mathrm{XL}$ ratio, and the cells in sub-G1 phase due to antitumoral drugs induced-cytotoxicity in human colon adenocarcinoma cells. Front Pharmacol. 2018;9:866.

11. de Lima Junior EA, et al. Doxorubicin caused severe hyperglycaemia and insulin resistance, mediated by inhibition in AMPk signalling in skeletal muscle. J Cachexia Sarcopenia Muscle. 2016;7(5):615-25.

12. Brady V, et al. Management of steroid-induced hyperglycemia in hospitalized patients with cancer: a review. Oncol Nurs Forum. 2014;41(6):E355-65.

13. Jafarpour-Sadegh F, et al. Consumption of fresh yellow onion ameliorates hyperglycemia and insulin resistance in breast cancer patients during doxorubicin-based chemotherapy: a randomized controlled clinical trial. Integr Cancer Ther. 2017;16(3):276-89. 